\title{
ON THE SUPERCENTER OF A GROUP OVER DOMAINS OF CHARACTERISTIC 0
}

\author{
HECTOR A. MERKLEN
}

\begin{abstract}
Let $G$ be a group and $R$ a commutative ring with 1 and let $U_{R} G$ be the group of units of the group ring $R G$. The $R$-supercenter of $G, S_{R} G$ is the set of elements of $G$ which have a finite number of conjugates under $U_{R} G$. The supercenter is studied in the case where $R$ is a domain of characteristic 0 . It is shown that for the most important cases the supercenter coincides with the center of the group and with the intersection of all group bases of the group ring.
\end{abstract}

1. Introduction. We use the following notations:

$G$ is a group, $T G$ the torsion of $G, F C(G)$ the $F C$-part of $G$;

$R$ is a commutative ring with $1 ; U_{R} G$ (resp. $V_{R} G$ ) is the group of units (resp. normalized units) of the group ring $R G$;

$F C_{R} G$ is the $F C$-part of $V_{R} G$;

$S_{R} G$, the $R$-supercenter of $G$, is the intersection of $G$ with $F C_{R} G$, i.e. the set of elements of $G$ which have a finite number of conjugates under $U_{R} G$.

We wish to investigate here the supercenter, $S_{R} G$, in the case where $R$ is an integral domain of characteristic 0 . The integral case, $S_{Z} G$, has been studied in [5] and the case of infinite fields, $S_{K} G$, in [3]. For the proofs we use some techniques, different from the ones used in those papers, which were introduced in [1] and [2]. Our Theorem 1 is a generalization of Theorem A of [3] (see Corollary 1).

Our results are the following:

THEOREM 1. Let $G$ be a torsion group.

(A) If $R$ has characteristic 0 , if $\{|g| \mid g \in G\} \cap U(R)=\{1\}$ and if the Jacobson radical of $R$ is different from 0 , then $T F C_{R} G=S_{R} G=Z(G)$.

(B) If $R$ contains a subring $R_{1}$ which is a domain of characteristic 0 with nonzero Jacobson radical, then $S_{R} G=Z(G)$.

COROLLARY 1. The supercenter of a torsion group over a field of characteristic 0 is equal to the center of the group.

THEOREM 2.

(A) Let $R$ be an integral domain such that no rational prime is a unit of $R$. Then, $T S_{R} G=T S$, where $S$ is the intersection of all group bases of $R G$.

(B) Let $G$ be a torsion group and let $R$ be an integral domain of characteristic 0 such that $\{|g| \mid g \in G\} \cap U(R)=\{1\}$. Then $S_{R} G=S$.

Received by the editors October 2, 1980.

1980 Mathematics Subject Classification. Primary 16A27.

(C) 1981 American Mathematical Society 0002-9939/81/0000-0403/\$01.75 
REMARK. It is easy to see that, for either case (A) or (B) in the theorems, the torsion of the $R$-supercenter of $G$ is normal in $U_{R} G$.

\section{Proofs.}

Theorem 1, (A). It suffices to show that if $x$ is a torsion element in $F C_{R} G$ then $x$ is in the center $Z(G)$ of $G$. We proceed by contradiction assuming that there is an element $t$ in $G$, of order $m$, which does not commute with $x$.

Since the support of $x$ is in $F C(G)$, this set together with $t$ generate a finite group. Hence, we can assume that $G$ is finite.

Let $r \neq 0$ be an element in the radical of $R$ and let us consider the unit $u=\sum_{i=0}^{m-1} r^{i} t^{i}$ with $u^{-1}=(1-r t)\left(1-r^{m}\right)^{-1}$. Then we must have that $[u, x]$ is a unit of finite order but this is a contradiction because the coefficient of 1 in $[u, x]$ is different from 0.

Theorem 1, (B). We reduce this to the previous case. We proceed as above and pick $r$ in the radical of $R_{1}$ such that either $r$ is integral over $Z$ or transcendent over $Q$. We require also that $r$ is a nonzero multiple of $n$, the order of $G=\langle x, t\rangle$.

If $D$ is the multiplicative subset formed by the elements of $Z[r]$ which are congruent to 1 modulo $r$, we introduce the ring $R^{\prime}=D^{-1}(Z[r]) \subset$.

In order that the proof for part (A) be applicable here, we only have to show that no proper divisor $d$ of $n$ is a unit of $R^{\prime}$. But, since $d^{-1}$ is of the form $P(r) /(1+Q(r) r)$, where $P, Q$ are polynomials with coefficients in $Z$, we deduce that, if $d$ is invertible in $R^{\prime}$, then it is invertible in $Z[r]$ and, therefore, $d=1$.

Theorem 2, proof that $T S \subset T S_{R} G$. For this part we do not need the stronger assumption about the units of $R$.

It suffices to show that if, for some prime $p, s$ is a $p$-element of $S$, then $s$ has a finite number of conjugates under $U_{R} G$. It is clear that these conjugates are also in $T S$ and then the lemma below says that they are obtained by conjugation by $G$. Hence, it will be enough to prove that for all $g$ in $G, g_{s g}^{-1} \in\langle s\rangle$.

If $v=\left(1+s+\cdots+s^{|s|-1}\right) \cdot g \cdot(1-s)$ we see that $u=1+v$ is a unit with inverse $1-v$ and, analyzing the relation $u s u^{-1} \in T S \subset G$, the claim follows.

LEMMA. Let $R$ have characteristic 0 and assume that $p$ is a rational prime which is not invertible in $R$. If $s, t$ are p-elements of $G$ which are conjugate under a unit $u$ of $U_{R} G$, then they are also conjugate under an element of $G$.

Proof. We proceed by contradiction, assuming that $s, t$ are not conjugate in $G$. Writing $u$ in the form $\Sigma \alpha_{g} g$, we have

$$
\sum \alpha_{g} g s=\sum \alpha_{g} t g .
$$

If $M_{\alpha}$ is the set of elements $g$ of $G$ which appear in (*) with coefficient $\alpha \neq 0$, we see that (*) defines a permutation $\sigma$, of $M_{\alpha}$, given by the identities $g s=t \sigma(g)$. If $\Gamma$, of length $k$, is an orbit of $M_{\alpha}$ under $\langle\sigma\rangle, \Gamma=\left\{g, \sigma(g), \ldots, \sigma^{k-1}(g)\right\}$, we have

$$
g s=t \sigma(g), \quad \sigma(g) s=t \sigma^{2}(g), \ldots, \sigma^{k-1}(g) s=t g
$$

and it is easy to see that $k$ is the least nonnegative integer such that $s^{k}=g^{-1} t^{k} g=$ $\left(g^{-1} t g\right)^{k}$. Our assumption that $s, t$ are not conjugate in $G$ implies then that $p$ divides 
$\alpha$. Since this is true for every $\alpha$, we get that $p$ divides the augmentation of $u$, a contradiction because $p$ is not invertible in $R$.

Theorem 2, proof that $T S_{R} G \subset T S$. Let us consider, as before, a p-element $s$ which now we assume to be a member of $S_{R} G$. Calling $G$ an arbitrary group basis of $R G$, we have to show that $s \in G$. Let us choose $t$ in $G$ as follows: in case (A), we apply [4, VI, 2.1] to pick $t$ a $p$-element in the support of $s$; in case (B), we choose any $t$ in the support of $s$. Then, $\left(s^{-1} t\right)^{|t|}$ is a product of conjugates of $s^{-1}$ and, therefore, has finite order. Hence, $s^{-1} t$ has finite order. Then, in case (A), we apply [4, II, 1.2] and, in case (B) [4, II, 1.4], and we obtain the desired conclusion: $s=t$.

Other immediate consequences of our results are

COROllary 2. For either case (A) or (B) of Theorem 2, if $N$ is a torsion, normal subgroup of $V_{R} G$, then $N$ is contained in the $R$-supercenter of $G$. (Use the techniques for the proof of Theorem 2.)

COROllary 3. Let $R$ contain a domain of characteristic 0 with nonzero Jacobson radical. Let $G$ be a torsion group and let us denote by $S$ the intersection of all group bases of $R G$. Then $S$ is contained in the center of $G$. If, furthermore, $R$ is a domain of characteristic 0 such that $\{|g| \mid g \in G\} \cap U(R)=1$, then $S=Z(G)$.

Proof. By the proof of Theorem $1, R$ contains $R^{\prime}$ which satisfies the hypotheses of Theorem 2, (B). Hence, $S_{R} G=Z(G)$ is the intersection of the group bases of $R^{\prime} G$. Since every group basis of $R^{\prime} G$ is a group basis of $R G, S \subset Z(G)$. On the other hand, if $R$ has the properties listed at the end, it is obvious that $Z(R) \subset S$.

\section{REFERENCES}

1. H. Merklen, $O$ grupo das unidades de um anel de grupo e o problema do isomorfismo, Tesse de Livre Docencia, Instituto de Matemática e Estatistica, Univ. de São Paulo, 1979.

2. H. Merklen and C. Polcino Milies, Group rings over $Z_{(p)}$ with FC unit groups, Canad. J. Math. (to appear).

3. C. Polcino Milies and S. K. Sehgal, FC elements in a group ring, Comm. Algebra (to appear).

4. S. K. Sehgal, Topics in group rings, Dekker, New York, 1978.

5. S. K. Sehgal and H. Zassenhaus, On the supercentre of a group and its ring-theoretic generalization (to appear).

Department of Mathematics, University of São Paulo, São Paulo, Brazil 